\title{
Dynamics of vibro-impact drilling with linear and nonlinear rock models
}

\author{
Maolin Liao ${ }^{\mathrm{a}}$, Yang Liu ${ }^{\mathrm{b}}$, Joseph Páez Chávez ${ }^{\mathrm{c}, \mathrm{d}}$, Antonio S.E. Chong ${ }^{\mathrm{c}, \mathrm{e}}$, Marian Wiercigroch ${ }^{\mathrm{e}}$ \\ ${ }^{a}$ School of Mechanical Engineering, University of Science and Technology Beijing, 100083, China \\ ${ }^{b}$ College of Engineering Mathematics and Physical Sciences, University of Exeter, North Park Road, Exeter EX4 $4 Q F$, UK \\ ${ }^{c}$ Facultad de Ciencias Naturales y Matemáticas, Escuela Superior Politécnica del Litoral, Km. 30.5 Vía Perimetral, P.O. \\ Box 09-01-5863, Guayaquil, Ecuador \\ ${ }^{d}$ Center for Dynamics, Department of Mathematics, TU Dresden, D-01062 Dresden, Germany \\ ${ }^{e}$ Centre for Applied Dynamics Research, School of Engineering, University of Aberdeen, Aberdeen AB24 $3 U E$, UK
}

\begin{abstract}
This paper presents a comprehensive numerical study of a higher order drifting oscillator that has been used to model vibro-impact drilling dynamics in previous publications by our research group [1-9]. We focus on the study of the bit-rock interactions, for which both linear and nonlinear models of the drilled medium are considered. Our investigation employed a numerical approach based on direct numerical integration via a newly developed MATLAB-based computational tool, ABESPOL [10], and based on path-following methods implemented via a software package for continuation and bifurcation analysis, COCO (Continuation Core) [11]. The analysis considered the excitation frequency, amplitude of excitation and the static force as the main control parameters, while the rate of penetration (ROP) was chosen as the main system output so as to assess the performance of the system when linear and nonlinear bitrock impact models are used. Furthermore, our numerical investigation reveals a rich system dynamics, owing to the presence of codimension-one bifurcations of limit cycles that influence the system behavior dramatically, as well as multistability phenomenon and chaotic motion.

Keywords: Vibro-impact drilling; Bit-rock interaction; Nonsmooth dynamical system; ABESPOL;

$\mathrm{COCO}$
\end{abstract}

\section{Introduction}

The development of innovative drilling techniques has attracted much attention during the past years, with studies focusing on both mathematical modeling and experimental research. In particular, special

Email addresses: mlin.liao@hotmail.com (Maolin Liao), y.liu2@exeter.ac.uk (Yang Liu), jpaez@espol.edu.ec (Joseph Páez Chávez), achong@espol.edu.ec (Antonio S.E. Chong), m.wiercigroch@abdn.ac.uk (Marian Wiercigroch) 
attention has been given to the study of rock deformation under static contact and dynamic impact, which can significantly influence the efficiency of the drilling process. Mishnaevsky [12] discussed optimal shapes of drilling tools, which turned out to be dependent on the type of drilled medium. For instance, cylindrical bits are most effective for brittle or quasi-brittle rocks, while conical bits are more convenient for hard plastic rocks. Cook et al. [13] conducted a series of experimental tests to observe hard rock deformation during loading and unloading phases. These experiments revealed that the elastic deformation of the rock formation is sustained for a certain period, until the applied load exceeds a threshold, which is dependent on the indenter's shape and size. In addition, the resulting penetration rate-load curves showed a linear dependence, except for the beginning of the loading phase and the end of the unloading phase.

In order to simulate elasto-plastic rock deformation, a number of bit-rock interaction models have been developed, such as Hertz [14], Maxwell [15], and Kelvin-Voigt [16] and others. Hertz model describes the rock deformation force by a nonlinear spring, where the nonlinear elastic coefficient of the spring is determined by the indenter's shape. Maxwell model considers a linear spring with a linear viscous damper in series, while the Kelvin-Voigt model combines them in parallel. Another approach is proposed by Wiercigroch's group [1], where they conducted a series of quasi-static rock deformation tests to explore the correlation between contact force and penetration rate [3]. In a subsequent work [2], they identified two clear regimes during the bit-rock interactions, namely, the loading and the unloading phases, which are characterized by different physical phenomena. In the loading phase, part of the input kinetic energy is dissipated due to friction, while the rest of the energy is stored as elastic strain energy. During the unloading phase, the stored elastic strain energy is released to recover a part of the rock indentation. The difference in the energy dissipation is reflected by the difference between the loading and unloading stiffness coefficients.

In the present work, we investigate in detail two types of bit-rock interaction models (linear and nonlinear) in a vibro-impact drilling system mimicking percussive drilling. Preliminary studies have been conducted by Sazidy et al. [17], who described the percussive drilling performance using a visco-elastoplastic rock model. Kovalyshen [18] carried out both simulations and experimental research using a drilling assembly with self-excited axial vibrations to observe the influence on the drilling performance. 
Han et al. [19] developed a model based on finite element analysis to describe the rock deformation during percussive drilling, finding also agreement with further experimental tests [20, 21]. In addition to [1-3], Wiercigroch and his co-workers have conducted extensive research in this area, both mathematical modelling and experimental studies. Ultrasonic percussive drilling is studied in detail in [22, 23], where the material removal rates are considered in [24]. A dry friction mechanism is used to build a model of percussive drilling [4], which is employed to explain the drop of the penetration rate under high static loads.

All bit-rock interaction models consider a set of parameters to describe the main physical characteristics of the drilled medium, and the precise values of such parameters deeply influences the overall system behaviour and performance. A bifurcation analysis of a low-dimensional percussive drilling model is presented in [25], which also considers the optimization of the penetration rates. In [16] a low-dimensional drifting oscillator is introduced for the first time to describe qualitatively the dynamics observed during percussive drilling. The analysis showed that the highest rates of penetration are achieved for period-one responses [5]. Based upon this work, they conducted a detailed bifurcation analysis of the system based on numerical continuation methods for nonsmooth dynamical systems [7], implemented via the software package TC-HAT [26]. In [27] an approach to reconstruct the oscillations of a vibro-impact is proposed, based on the strategy employed in [6]. These works considered both bifurcation techniques [8] and contact duration methods [9] to estimate the stiffness of the impacted medium in the framework of percussive drilling.

In order to explore bifurcation scenarios of the vibro-impact drilling system having linear and nonlinear bit-rock interaction laws, a combination of numerical tools based on direct numerical integration and path-following methods will be used in the present work. For this purpose, we will employ the software package COCO, developed by Dankowicz and Schilder [11]. COCO is a MATLAB-based platform that provides standard functionality required for investigating bifurcations. In addition to COCO we will employ the recently developed computational suite ABESPOL [10], which implements a set of numerical routines used to analyze dynamical systems. For instance, the software ABESPOL provides in a semi automatic manner time histories, Poincaré maps, bifurcation diagrams and basins of attraction, among 
other dynamical objects. The thus obtained numerical data can then be used to start the path-following analysis using COCO, as has been done in e.g. [10, 28, 29], and this approach will be applied in the present work.

The paper is organized as follows. In Section 2, the mathematical modeling of a bit-rock vibro-impact system is introduced. Here, we also carry out a comparison of the linear and nonlinear bit-rock interaction models taking into account energy dissipation. In addition, a suitable linear transformation is introduced in order to decouple the periodic behavior from the drift [6]. Section 3 is devoted to the investigation of the dynamical response of the vibro-impact system, under both linear and nonlinear bit-rock interactions. The numerical study based on direct numerical integration and path-following methods is conducted via the software ABESPOL, as explained above. The effect on the penetration rate under linear and nonlinear bit-rock impact models is studied in detail in Section 4. To finish, concluding remarks and outlook for future work is provided in Section 5.

\section{Mathematical modeling}

Fig. 2.1 (a) presents the physical model of the vibro-impact drilling system to be analyzed in the present work, which has been previously proposed in [9,30]. The model consists of a mass $m_{2}$ that represents the drill-bit assembly is excited by a sinusoidal force with amplitude $F_{a}$ and frequency $\Omega$. This mass is connected to the mass $m_{1}$ which stands for the drill-string components above the drill-bit assembly. $F_{b}$ accounts for the static force applied to $m_{1}$ in drilling direction, and $m_{1}$ and $m_{2}$ are coupled via a spring with stiffness $k$ and a damper with coefficient $c . G$ is the initial gap between the bit and the rock surface represented by the plate of the slider. The interactions between the drill-bit and the rock surface can be modeled using the linear (solid lines) or nonlinear (dash lines) interaction models as shown in Fig. 2.1 (b), where $X_{p}, X_{f}, X^{*}$, and $F^{*}$ are the initial position of the drill bit when it contacts rock surface, the end position of the drill bit when it leaves rock surface, the maximal bit penetration in drilled medium, and the maximal bit-rock force, respectively. It should be noted that the end positions of the linear and nonlinear interaction models, $X_{f}^{l i n}$ and $X_{f}^{n l i n}$, when the drill bit leaves the rock medium, are different. In addition, we assume that the energy dissipations of both models for an entire interaction 
are equivalent, i.e. the light grey area shown in Fig. 2.1 (b) equals to the dark grey one.

(a)

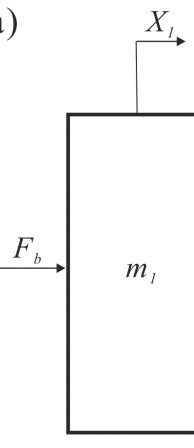

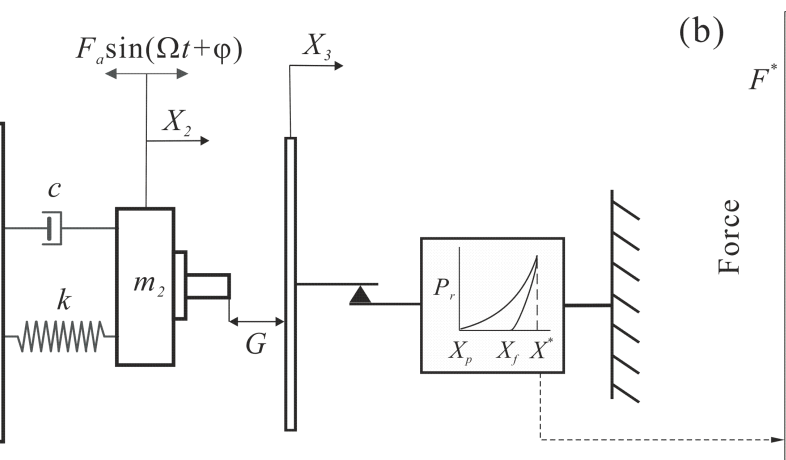

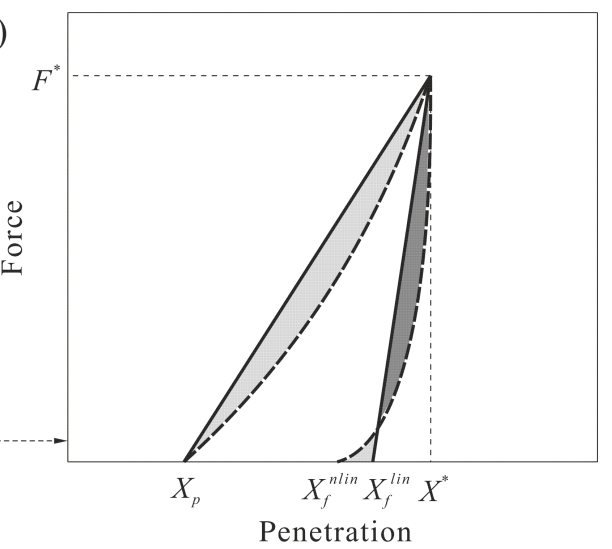

Figure 2.1: (a) Physical model of the vibro-impact drilling system [30], where $X_{1}, X_{2}$, and $X_{3}$ stand for the absolute positions of $m_{1}, m_{2}$, and the plate of the slider, respectively. An initial gap $G$ is set between the bit and the rock surface. (b) Linear (solid lines) and nonlinear (dash lines) bit-rock interaction models. In this model, the end positions of the linear $\left(X_{f}^{l i n}\right)$ and nonlinear $\left(X_{f}^{n l i n}\right)$ interaction models are different, while the energy dissipations of both models are equivalent, i.e. the light grey area equals to the dark grey area.

\subsection{Linear and nonlinear bit-rock interaction models}

As shown in Fig. 2.1 (b), the energy dissipation of the linear bit-rock model can be calculated as,

$$
E_{l i n}=\frac{1}{2} k_{l}^{l i n}\left(X^{*}-X_{p}\right)^{2}-\frac{1}{2} k_{u}^{l i n}\left(X^{*}-X_{f}^{l i n}\right)^{2},
$$

where $k_{l}^{l i n}, k_{u}^{\text {lin }}$ are the loading and unloading stiffnesses of the linear model. The energy dissipation for the nonlinear bit-rock model can be calculated as,

$$
E_{n l i n}=\int_{X_{p}}^{X^{*}} k_{l}^{n l i n}\left(X-X_{p}\right)^{n_{l}} \mathrm{~d} X-\int_{X_{f}^{n l i n}}^{X^{*}} k_{u}^{n l i n}\left(X-X_{f}^{n l i n}\right)^{n_{u}} \mathrm{~d} X
$$

where $k_{l}^{\text {nlin }}, k_{u}^{\text {nlin }}$ are the loading and unloading stiffnesses of the nonlinear model. For a complete bit-rock interaction, if the energy dissipations of these two models are equivalent, it gives

$$
E_{\text {lin }}=E_{n l i n} .
$$


In Eq. (2.1), according to the material experiments in [9], $X_{p}, X_{f}^{n l i n}, X^{*}$, and $F^{*}$ can be measured, $k_{l}^{\text {lin }}$, $k_{l}^{n l i n}, k_{u}^{n l i n}, n_{l}$, and $n_{u}$ can be determined using experimental data, and $X_{f}^{l i n}$ and $k_{u}^{\text {lin }}$ can be calculated using

$$
F^{*}=k_{u}^{l i n}\left(X^{*}-X_{f}^{l i n}\right) .
$$

Therefore, by adopting this approach, all the bit-rock interactive parameters for the linear and nonlinear models can be obtained. A detailed consideration of two possible operating regimes are given below.
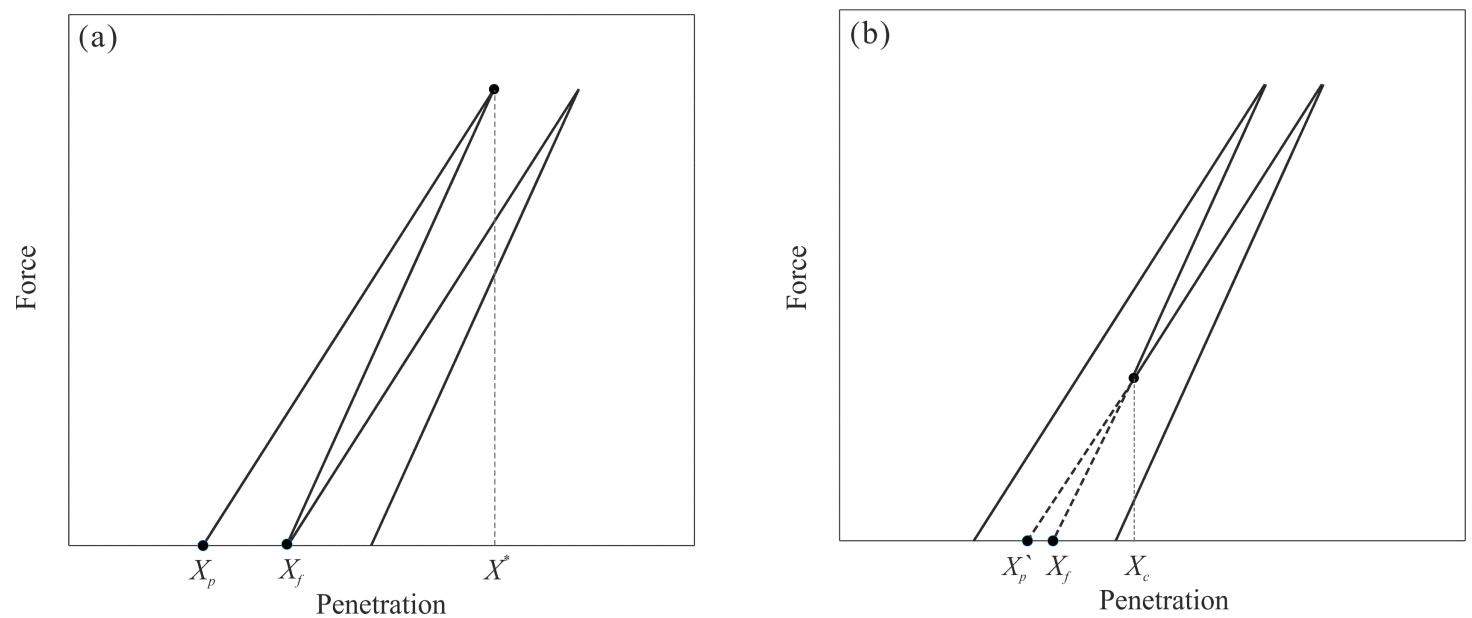

Figure 2.2: Bit-rock impact regimes: (a) one impact and (b) multiple impacts.

As shown in Fig. 2.2, both linear and nonlinear bit-rock models can be operated in one-impact or multiple-impact regime. When the drill bit is in one-impact regime, the end position of the unloading phase $X_{f}$ can be calculated using $[1,2]$

$$
X_{f}=X^{*}-\left[\frac{k_{l}\left(X^{*}-X_{p}\right)^{n_{l}}}{k_{u}}\right]^{\frac{1}{n_{u}}},
$$

once the maximal progression $X^{*}$ is achieved. Then the calculated $X_{f}$ can be used as the starting position of the next impact period. When the drill bit is in multiple-impact regime as shown in Fig. 2.2 (b), successive loading contact occurs at $X_{c}$, and its auxiliary starting position for the next loading phase $X_{p}^{\prime}$ can be calculated as

$$
X_{p}^{\prime}=X_{c}-\left[\frac{k_{u}\left(X_{c}-X_{f}\right)^{n_{u}}}{k_{l}}\right]^{\frac{1}{n_{l}}}
$$


It is worth noting that the calculated $X_{p}^{\prime}$ should be less than the predicted end position of the previous impact period $X_{f}$.

\subsection{Equations of motion}

According to the physical model shown in Fig. 2.1, the motion of the vibro-impact drilling system can be divided into three modes: no contact, loading contact, and unloading contact.

No contact. This mode occurs when $X_{2}-X_{3}<G$, i.e. the drill-bit is not in contact with the drilled medium, and the dynamics of the system is governed by

$$
\left\{\begin{array}{l}
m_{1} \ddot{X}_{1}+c\left(\dot{X}_{1}-\dot{X}_{2}\right)+k\left(X_{1}-X_{2}\right)=F_{b} \\
m_{2} \ddot{X}_{2}+c\left(\dot{X}_{2}-\dot{X}_{1}\right)+k\left(X_{2}-X_{1}\right)=F_{a} \sin \left(\Omega t+\varphi_{0}\right) \\
\dot{X}_{3}=0 \\
\dot{X}_{p}=0 \\
\dot{X}_{f}=0
\end{array}\right.
$$

This operation mode terminates when $m_{2}$ hits the slider $\left(X_{2}=X_{3}+G\right)$, and the system switches to the loading contact mode. The last two equations are auxiliary ODEs whose purpose is to add the values $X_{p}$ and $X_{f}$ (see Fig. 2.1) in the state variables, which is done only for the sake of the numerical implementation in ABESPOL. As will be seen later, $X_{p}$ and $X_{f}$ will vary in an impulsive manner via suitably defined jump functions, according to the contact model described in the preceding sections.

Loading contact. This regime takes place when the mass $m_{2}$ is in contact with the slider $\left(X_{2}=X_{3}+G\right)$ 
and the mass moves rightwards $\left(\dot{X}_{2}>0\right)$. The response of the system can be described as

$$
\left\{\begin{array}{l}
m_{1} \ddot{X}_{1}+c\left(\dot{X}_{1}-\dot{X}_{2}\right)+k\left(X_{1}-X_{2}\right)=F_{b} \\
m_{2} \ddot{X}_{2}+c\left(\dot{X}_{2}-\dot{X}_{1}\right)+k\left(X_{2}-X_{1}\right)+k_{l}\left(X_{2}-X_{p}-G\right)^{n_{l}}=F_{a} \sin \left(\Omega t+\varphi_{0}\right) \\
\dot{X}_{3}=\dot{X}_{2} \\
\dot{X}_{p}=0 \\
\dot{X}_{f}=0
\end{array}\right.
$$

This operation mode terminates when $m_{2}$ changes direction, i.e., $\dot{X}_{2}\left(t_{\mathrm{f}}\right)=0$ and $\ddot{X}_{2}\left(t_{\mathrm{f}}\right) \neq 0$. At this moment, the impulsive variation takes place

$$
X_{f}\left(t_{\mathrm{f}}^{+}\right)=X_{2}\left(t_{\mathrm{f}}^{-}\right)-G-\left(\frac{k_{l}}{k_{u}}\right)^{\frac{1}{n_{u}}}\left(X_{2}\left(t_{\mathrm{f}}^{-}\right)-X_{p}\left(t_{\mathrm{f}}^{-}\right)-G\right)^{\frac{n_{l}}{n_{u}}},
$$

where $X_{2}\left(t_{\mathrm{f}}^{-}\right)$gives the maximum attained value of $X_{2}$ before it changes direction. The formula above determines the value of $X_{f}$ to be used for the unloading mode (see below).

Unloading contact. Similarly to the previous regime, the unloading mode occurs when $m_{2}$ is in contact with the slider $\left(X_{2}=X_{3}+G\right)$, only that now the mass is moving leftwards $\left(\dot{X}_{2}<0\right)$ and $X_{3}>X_{f}$. During this regime, the dynamics of the vibro-impact system is determined by

$$
\left\{\begin{array}{l}
m_{1} \ddot{X}_{1}+c\left(\dot{X}_{1}-\dot{X}_{2}\right)+k\left(X_{1}-X_{2}\right)=F_{b} \\
m_{2} \ddot{X}_{2}+c\left(\dot{X}_{2}-\dot{X}_{1}\right)+k\left(X_{2}-X_{1}\right)+k_{u}\left(X_{2}-X_{f}-G\right)^{n_{u}}=F_{a} \sin \left(\Omega t+\varphi_{0}\right) \\
\dot{X}_{3}=\dot{X}_{2} \\
\dot{X}_{p}=0 \\
\dot{X}_{f}=0
\end{array}\right.
$$

This operation mode can terminate in two forms. The first one is that, when $X_{3}\left(t_{\mathrm{f}}\right)=X_{f}\left(t_{\mathrm{f}}\right), m_{2}$ loses 
contact with the slider, and the system switches to the no contact mode with the impulsive variation

$$
X_{p}\left(t_{\mathrm{f}}^{+}\right)=X_{3}\left(t_{\mathrm{f}}^{-}\right) .
$$

This resets $X_{p}$ to the final slider displacement $X_{3}$. The second way to terminate this regime occurs when $m_{2}$ changes direction, i.e., $\dot{X}_{2}\left(t_{\mathrm{f}}\right)=0$ and $\ddot{X}_{2}\left(t_{\mathrm{f}}\right) \neq 0$. At this moment, the system goes back to the loading contact mode, with the impulsive variation

$$
X_{p}\left(t_{\mathrm{f}}^{+}\right)=X_{2}\left(t_{\mathrm{f}}^{-}\right)-G-\left(\frac{k_{u}}{k_{l}}\right)^{\frac{1}{n_{l}}}\left(X_{2}\left(t_{\mathrm{f}}^{-}\right)-X_{f}\left(t_{\mathrm{f}}^{-}\right)-G\right)^{\frac{n_{u}}{n_{l}}} .
$$

This assigns the value of $X_{p}$ to be used for the contact force during the loading mode, in such a way that the penetration-force curve is continuous during operation.

\subsection{Nondimensionalization and decoupling of the system drift}

In this study, we will use the following dimensionless variables and parameters:

$$
\begin{aligned}
& x_{1}=\frac{X_{1}}{\mu}, \\
& x_{2}=\frac{X_{2}}{\mu}, \\
& x_{3}=\frac{X_{3}}{\mu}, \\
& x_{p}=\frac{X_{p}}{\mu}, \\
& x_{f}=\frac{X_{f}}{\mu}, \\
& \Omega_{0}=\sqrt{\frac{k_{l} \mu^{n_{l}-1}}{m_{2}}}, \\
& \tau=\Omega_{0} t, \\
& \omega=\frac{\Omega}{\Omega_{0}}, \\
& \gamma=\frac{m_{2}}{m_{1}}, \\
& a=\frac{F_{a}}{m_{2} \mu \Omega_{0}^{2}}, \\
& b=\frac{F_{b}}{m_{2} \mu \Omega_{0}^{2}}, \\
& \beta=\frac{k \mu^{1-n_{l}}}{k_{l}}, \\
& \xi=\frac{c}{2 m_{2} \Omega_{0}}, \\
& g=\frac{G}{\mu}, \\
& \kappa=\frac{k_{u} \mu^{n_{u}-n_{l}}}{k_{l}} .
\end{aligned}
$$

In order to apply numerical continuation techniques for periodic solutions, the drifting motion of the vibro-impact drilling system should be separated from the periodic behaviour of the model. By adopting the transformation proposed by Pavlovskaia et al. [6], we introduce the change of coordinates as follows:

$$
\left\{\begin{array}{l}
z_{1}=x_{1}, \\
z_{2}=x_{2}-x_{1}, \\
z_{3}=x_{3}-x_{1}, \\
z_{p}=x_{p}-x_{1}, \\
z_{f}=x_{f}-x_{1} .
\end{array}\right.
$$


Apply this coordinate changes and the nondimensionalization, we can obtain the following transformed model for the vibro-impact system.

No contact $\left(z_{2}-z_{3}<g\right)$ :

$$
\left\{\begin{array}{l}
z_{1}^{\prime \prime}-2 \xi \gamma z_{2}^{\prime}-\beta \gamma z_{2}=b \gamma \\
z_{2}^{\prime \prime}+2 \xi(\gamma+1) z_{2}^{\prime}+\beta(\gamma+1) z_{2}=a \sin \left(\omega \tau+\varphi_{0}\right)-b \gamma \\
z_{3}^{\prime}=-z_{1}^{\prime} \\
z_{p}^{\prime}=-z_{1}^{\prime} \\
z_{f}^{\prime}=-z_{1}^{\prime}
\end{array}\right.
$$

Loading contact $\left(z_{2}=z_{3}+g\right.$ and $\left.z_{2}^{\prime}+z_{1}^{\prime}>0\right)$ :

$$
\left\{\begin{array}{l}
z_{1}^{\prime \prime}-2 \xi \gamma z_{2}^{\prime}-\beta \gamma z_{2}=b \gamma \\
z_{2}^{\prime \prime}+2 \xi(\gamma+1) z_{2}^{\prime}+\beta(\gamma+1) z_{2}+\left(z_{2}-z_{p}-g\right)^{n_{l}}=a \sin \left(\omega \tau+\varphi_{0}\right)-b \gamma \\
z_{3}^{\prime}=z_{2}^{\prime} \\
z_{p}^{\prime}=-z_{1}^{\prime} \\
z_{f}^{\prime}=-z_{1}^{\prime}
\end{array}\right.
$$

with the impulsive variation

$$
z_{f}\left(t_{\mathrm{f}}^{+}\right)=z_{2}\left(t_{\mathrm{f}}^{-}\right)-g-\kappa^{-\frac{1}{n_{u}}}\left(z_{2}\left(t_{\mathrm{f}}^{-}\right)-z_{p}\left(t_{\mathrm{f}}^{-}\right)-g\right)^{\frac{n_{l}}{n_{u}}},
$$

when $z_{2}^{\prime}\left(t_{\mathrm{f}}\right)+z_{1}^{\prime}\left(t_{\mathrm{f}}\right)=0$. 
Unloading contact $\left(z_{2}=z_{3}+g, z_{2}^{\prime}+z_{1}^{\prime}<0\right.$, and $\left.z_{3}>z_{f}\right)$ :

$$
\left\{\begin{array}{l}
z_{1}^{\prime \prime}-2 \xi \gamma z_{2}^{\prime}-\beta \gamma z_{2}=b \gamma \\
z_{2}^{\prime \prime}+2 \xi(\gamma+1) z_{2}^{\prime}+\beta(\gamma+1) z_{2}+\kappa\left(z_{2}-z_{f}-g\right)^{n_{u}}=a \sin \left(\omega \tau+\varphi_{0}\right)-b \gamma \\
z_{3}^{\prime}=z_{2}^{\prime} \\
z_{p}^{\prime}=-z_{1}^{\prime} \\
z_{f}^{\prime}=-z_{1}^{\prime} .
\end{array}\right.
$$

with the impulsive variation

$$
z_{p}\left(t_{\mathrm{f}}^{+}\right)=z_{3}\left(t_{\mathrm{f}}^{-}\right),
$$

when $z_{3}\left(t_{\mathrm{f}}\right)=z_{f}\left(t_{\mathrm{f}}\right)$ and

$$
z_{p}\left(t_{\mathrm{f}}^{+}\right)=z_{2}\left(t_{\mathrm{f}}^{-}\right)-g-\kappa^{\frac{1}{n_{l}}}\left(z_{2}\left(t_{\mathrm{f}}^{-}\right)-z_{f}\left(t_{\mathrm{f}}^{-}\right)-g\right)^{\frac{n_{u}}{n_{l}}},
$$

in case $z_{2}^{\prime}\left(t_{\mathrm{f}}\right)+z_{1}^{\prime}\left(t_{\mathrm{f}}\right)=0$.

\section{Numerical investigations of system dynamics}

For the vibro-impact drilling system, the frequency and amplitude of excitation, and the static force are the main control parameters to improve the stability and efficiency of the drilling process. This section will consider the influence of these three control parameters on the dynamics of the drilling system, and compare the linear and nonlinear bit-rock interaction models through numerical integration and continuation.

\subsection{Bifurcation analysis}

\subsubsection{Influence of excitation frequency}

The studied range of the nondimensional frequency was set for $\omega \in[0.05,0.4]$, and the numerical results through direct integration and continuation for the vibro-impact drilling system with the linear interaction 
model are shown in Fig. 3.1, where bifurcations for both increasing and decreasing the frequency of excitation are presented. Co-existing attractors are marked by red dots, and these co-existing orbits are plotted on the phase plane $\left(z_{2}, v_{2}\right)$ as shown in Fig. 3.1 (c-h). According to our calculations, three coexisting regimes $\left(I_{1}, C_{1}\right.$, and $\left.I_{2}\right)$ were identified as presented in Fig. 3.1 (a). The first co-existing regime $\left(I_{1}\right)$ was observed for $\omega \in[0.095,0.101]$ at where a period-two response with four impacts per period of excitation co-exists with a period-one response with one impact. The co-existence of a chaotic response and a quasi-periodic response for the second regime $\left(C_{1}\right)$ was recorded for $\omega \in[0.102,0.121]$. In the third regime $\left(I_{2}\right), \omega \in[0.3759,0.4]$, a period-two response with two impacts per period of excitation co-exists with a period-two response with one impact. In addition to direct integration, numerical continuation was carried out, saddle-node, period-doubling, and Neimark-Sacker bifurcations were found in the given frequency range. It can be seen from Fig. 3.1 (b) that, as the frequency increases, a saddle-node bifurcation appears at $\omega=0.0771$ which terminates the chaotic motions existed for $\omega<0.0771$. Then, a pair of Neimark-Sacker bifurcations can be observed at $\omega=0.0785$ and $\omega=0.0871$ followed by a perioddoubling cascade leading to chaos. In the decreasing direction of frequency, the period-one response with one impact encounters a pair of Neimark-Sacker bifurcations at $\omega=0.1449$ and $\omega=0.1010$. The extension of the stable orbit towards the left and the right side is terminated by a saddle-node bifurcation at $\omega=0.0950$ and a period-doubling bifurcation at $\omega=0.3064$, respectively. In addition, another saddlenode bifurcation which comes from the co-existing period-two response with one impact is recorded at $\omega=0.3759$.

Bifurcation diagrams using the nondimensional frequency as a branching parameter for the vibroimpact drilling system with the nonlinear interaction model are shown in Fig. 3.2. Comparing to the model with the linear interaction model, the chaotic regime for the nonlinear one is shrunk to a low frequency range constrained by a saddle-node bifurcation at $\omega=0.1058$. For the nonlinear model, only one co-existing regime, $\omega \in[0.127,0.138]$, is observed where a period-two response with four impacts co-exists with a period-one response with one impact. The period-two response then bifurcates into a chaotic response through a series of period-doubling bifurcations co-existing with the period-one response until $\omega=0.138$. For the linear interaction model, the period-one response bifurcates into a period-two 
(a)

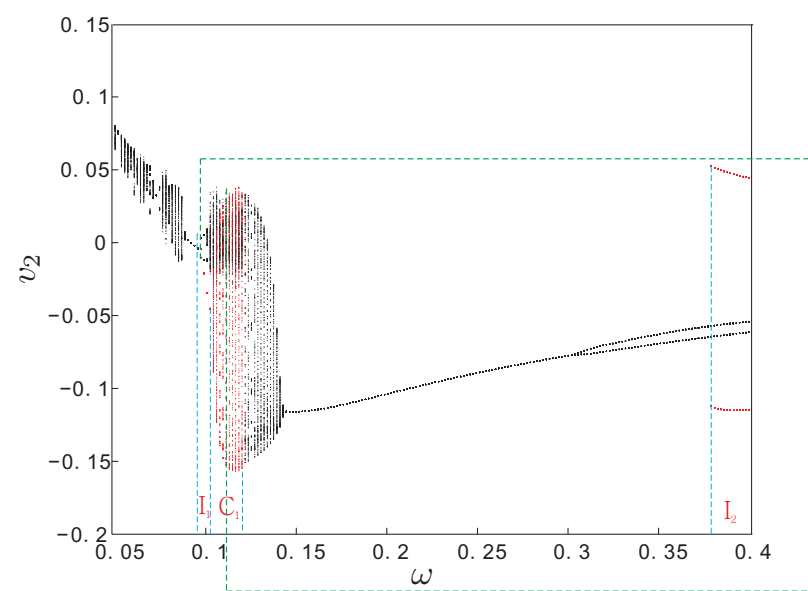

(b)

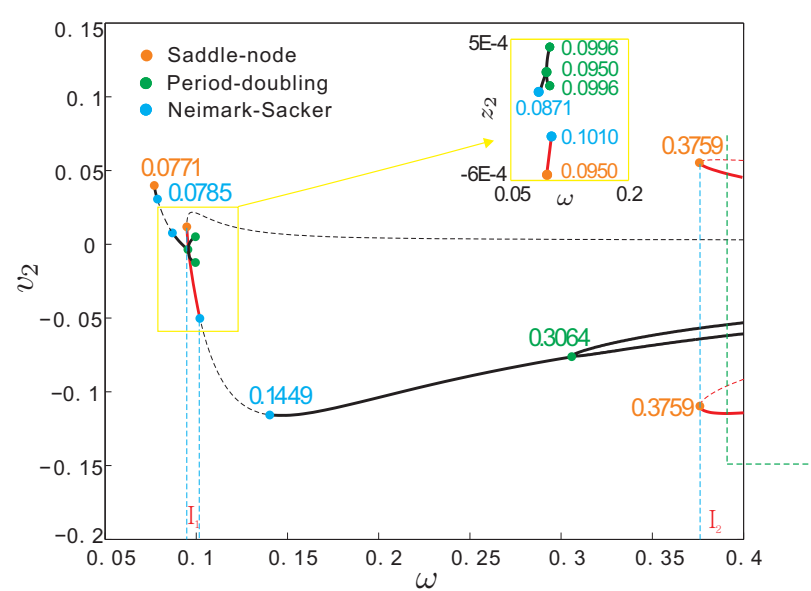

(c)

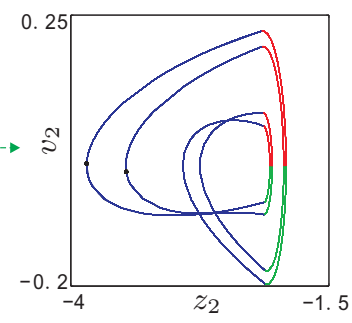

(d)

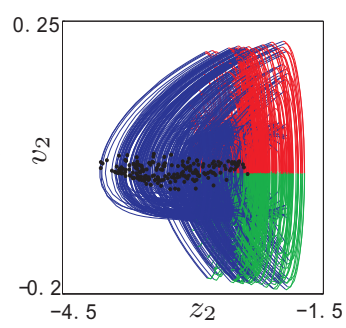

(e)

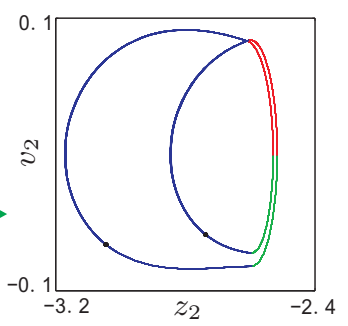

(f)

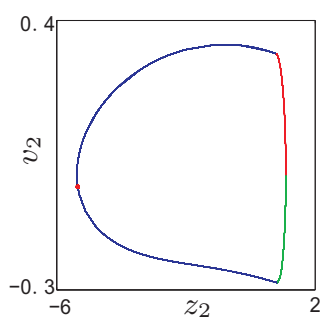

(g)

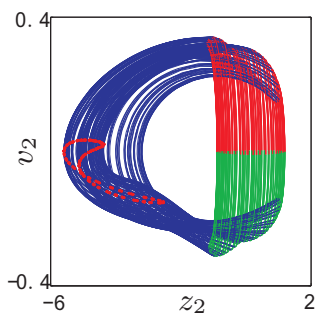

(h)

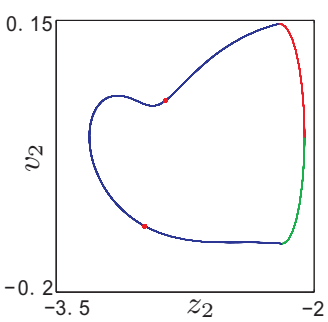

Figure 3.1: Bifurcation diagrams of the nondimensional frequency for the linear interaction model calculated for $a=0.0130$, $b=0.0093, \xi=0.0172, \alpha=0.0133, \beta=0.0033, g=0.02, \omega \in[0.05,0.4], n_{1}=1, n_{u}=1$, and $\kappa=1.1706$. Subplot (a) shows the result of numerical integration and subplot (b) shows the result of numerical continuation. In subplot (a), the black and red dots represent the sweeping of frequency from increasing and decreasing direction, respectively. In subplot (b), solid and dashed segments represent stable and unstable branches, respectively. Saddle-node, period-doubling, and Neimark-Sacker bifurcations are marked by orange, green, and blue dots, respectively. The segments in the yellow boxes are plotted using $z_{2}$ as the y-axis to verify that the intersection between the red and black branches is caused by projection. The frequency ranges $I_{1}$ and $I_{2}$ show co-existing stable attractors, and the frequency window $C_{1}$ has co-existing chaotic and quasi-periodic attractors. Subplots (c),(d),(e) display the phase trajectories for $\omega=0.098,0.113$, and 0.393, respectively, and their corresponding co-existing trajectories are shown in the subplots $(\mathrm{f}),(\mathrm{g}),(\mathrm{h})$, where blue, red, green segments indicate no contact, loading contact, and unloading contact phases, respectively.

response with two impacts per period of excitation via a period-doubling at $\omega=0.3064$ as shown in Fig. 3.1

(b), while for the nonlinear one, this period-doubling bifurcation occurs at $\omega=0.3795$ as presented in

Fig. 3.2 (b). Therefore, the frequency range for the period-one response with one impact is enlarged from $\omega \in[0.0950,0.3064]$ for the linear model to $\omega \in[0.127,0.3795]$ for the nonlinear model, which provides a better stability for vibro-impact drilling. 
(a)

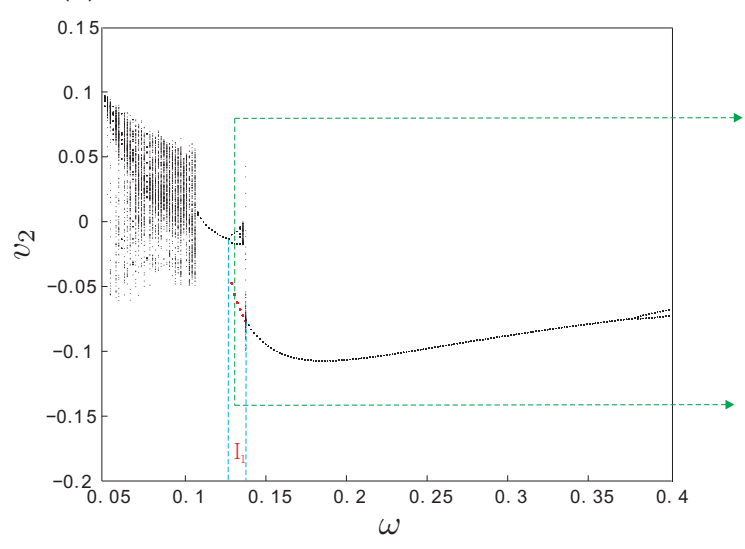

(b)

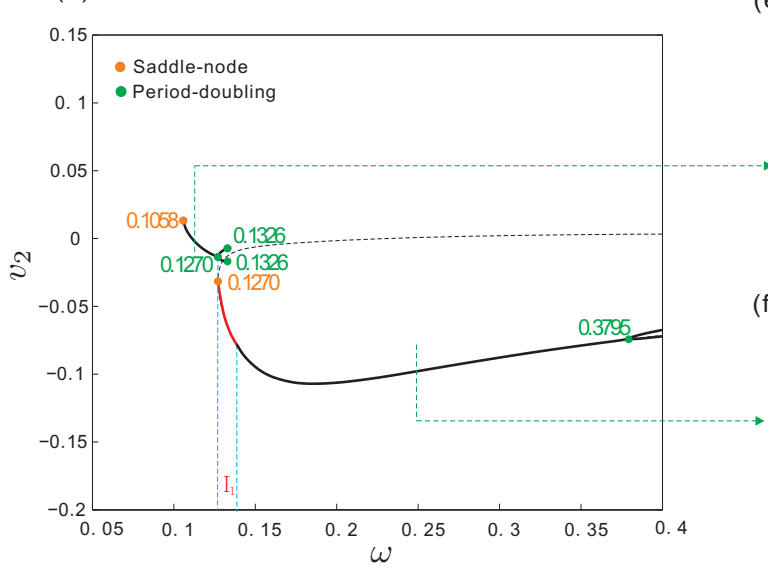

(c)

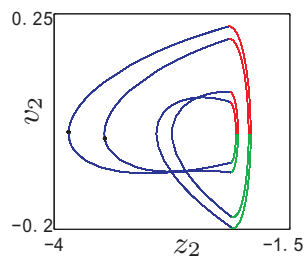

(d)

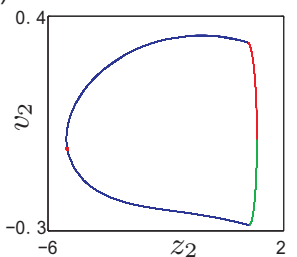

(e)

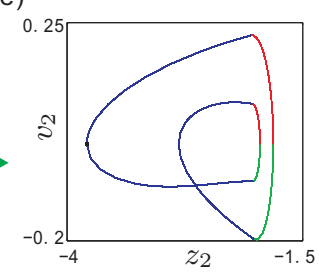

(f)

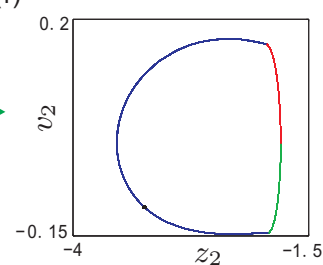

(g)

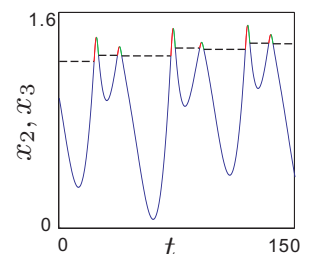

(h)

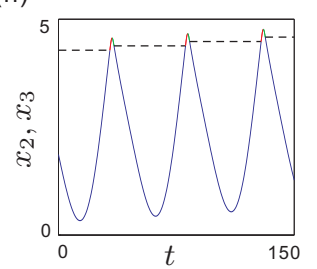

(i)

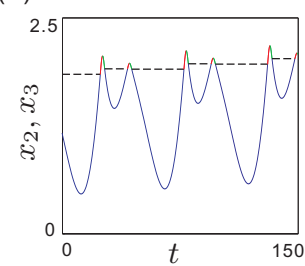

(j)

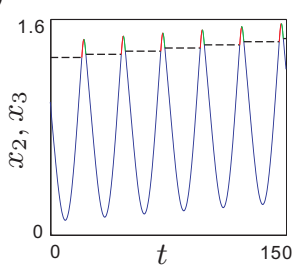

Figure 3.2: Bifurcation diagrams of the nondimensional frequency for the nonlinear interaction model calculated for $a=$ $0.0159, b=0.0114, \xi=0.0188, \alpha=0.0133, \beta=0.0040, g=0.02, \omega \in[0.05,0.4], n_{1}=0.86, n_{u}=1.31$, and $\kappa=$ 3.1815. Subplots (a) and (b) show the results of numerical integration and numerical continuation, respectively. Co-existing attractors at $\omega=0.1305$ are shown in subplots (c) and (d). Subplot (e) displays a period-one response with two impacts at $\omega=0.118$, and subplot (f) presents a period-one response with one impact at $\omega=0.250$. Subplots (g)-(j) display the time series of the displacements of drill bit (solid lines) and rock surface (dashed lines) corresponding to subplots (c)-(f).

\subsubsection{Influence of excitation amplitude}

In this subsection, the nondimensional amplitude of external excitation is studied as a bifurcation parameter, and the parameter range is set for $a \in[0,0.04]$. For the linear and nonlinear interaction models, both numerical integration and continuation were carried out, and their results are shown in Fig. 3.3. It can be seen from the figure that the period-one responses for the linear and nonlinear interaction models emerge after a saddle-node bifurcation at $a=0.0047$ and $a=0.0075$, respectively. The first period-doubling for the system with the linear model occurs at $a=0.157$, and the second perioddoubling is recorded at $a=0.0184$ followed by a period-doubling cascade from $a=0.0191$. For the system 
with the nonlinear model, the first period-doubling happens at $a=0.0213$, which is followed by the second and the period-doubling cascade at $a=0.0253$ and $a=0.0264$, respectively. As the amplitude increases, both period-doubling cascades lead the system to chaos. Comparing both bifurcation scenarios, it can be found that their bifurcation structures are similar, but the regime of the stable period-one response for the nonlinear model $(a \in[0.0075,0.0213])$ is larger than the linear one $(a \in[0.0047,0.0157])$. In addition, the nonlinear model has two small periodic windows of period-three responses with two impacts per period of excitation. It is worth noting that the multiple-impact regime studied using Eq. (2.1) can be observed from Fig. 3.3 (f) and (k). From the point of view of system response, the system with the nonlinear interaction model has larger stable regime than the linear one when the amplitude of external excitation is small, while the system responses under both interaction models are similar. Therefore, under small amplitude of excitation, the linear bit-rock interaction model can predict the dynamics of vibro-impact drilling effectively.

\subsubsection{Influence of static force}

The influences of the nondimensional static force on system dynamics are studied in this subsection, and the simulation results calculated using numerical integration and continuation for the vibro-impact drilling system with the linear and nonlinear interaction models are shown in Fig. 3.4. Comparing the bifurcation diagrams obtained by numerical integration, both systems have chaotic motions when static force is small. As static force increases, the system with the linear model experiences a reverse period-doubling cascade from $b=0.0063$ leading to period-one motion with one impact at $b=0.0077$. The system with the nonlinear model has a small window of period-two motion with one impact in $b \in[0.0054,0.0056]$, and its reverse period-doubling is encountered from $b=0.0073$ leading to period-one motion with one impact per period of excitation at $b=0.0088$. The period-one responses for the linear and nonlinear model terminate at $b=0.0257$ and $b=0.0203$ via saddle-node bifurcation, respectively. Comparing the simulation results calculated by numerical continuation, the basic structures of their bifurcations are similar. The regime for the stable period-one response with one impact per period of excitation obtained by the linear interaction model $(b \in[0.0077,0.0257])$ is larger than the one obtained 
(a)

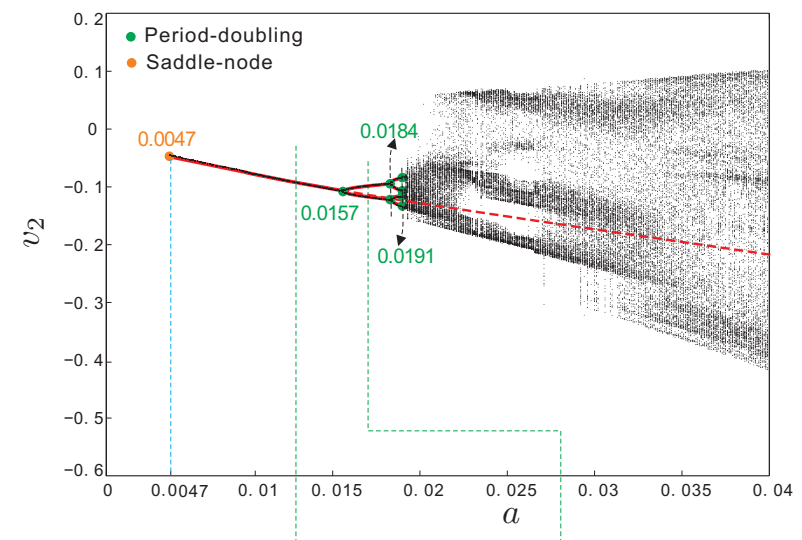

(b)

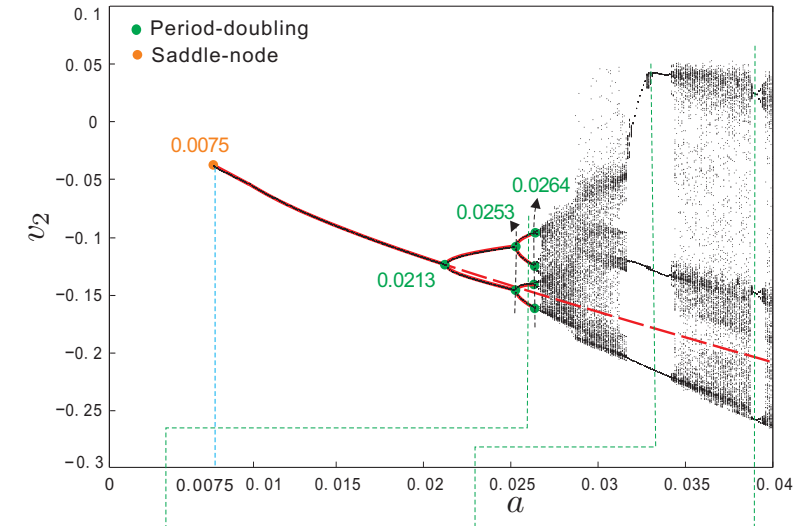

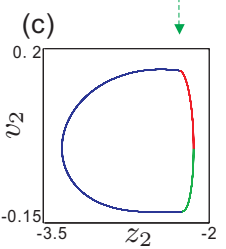

(h)

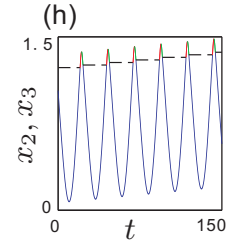

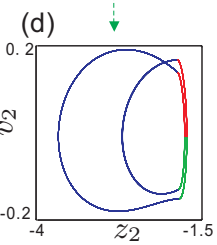

(i)

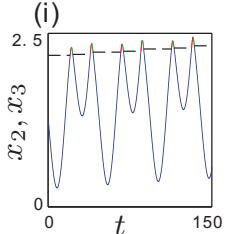

(e)
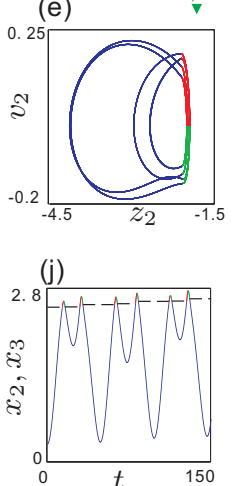

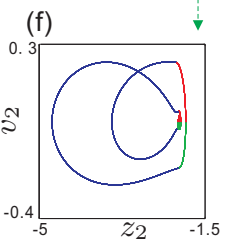

$(\mathrm{k})$

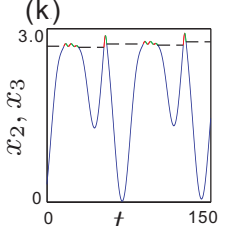

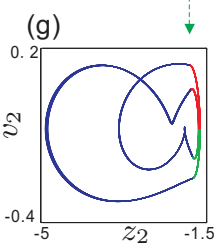

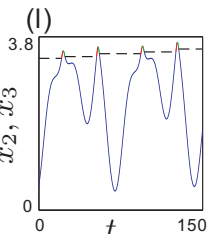

Figure 3.3: Bifurcation diagrams of the nondimensional amplitude of the higher order drifting oscillator with the (a) linear and (b) nonlinear impact models, where the nondimensional parameters for subplot (a) are $a \in[0.0047,0.04], b=0.0093$, $\xi=0.0172, \alpha=0.0133, \beta=0.0033, g=0.02, \omega=0.2353, n_{1}=1, n_{u}=1$, and $\kappa=1.1706$, and the equivalent parameters for subplot (b) are $a \in[0.0075,0.04], b=0.0114, \xi=0.0188, \alpha=0.0133, \beta=0.0040, g=0.02, \omega=0.2595, n_{1}=0.86$, $n_{u}=1.31$, and $\kappa=3.1815$. The results of numerical integration are shown by black dots, while the stable and unstable branches obtained by numerical continuation are denoted by solid and dashed red lines. Subplots (c)-(g) and (h)-(l) display the phase trajectories and time series of the periodic responses, respectively.

by the nonlinear model $(b \in[0.0088,0.0203])$. In addition, at $b=0.0054$, a Neimark-Sacker bifurcation is detected for the nonlinear model leading the system to a period-two motion with one impact per period of excitation, and this periodic motion is terminated by a saddle-node bifurcation at $b=0.0056$.

\subsection{Two-parameter continuation}

This subsection will study two-parameter continuation of the higher order drifting oscillator using COCO through exploring codimension-one bifurcations, such as saddle-node bifurcation and perioddoubling bifurcation, and then the traces of these bifurcations will be extended to two-parameter space. As shown in Fig. 3.5 (a)-(d), the orange and green bifurcation curves are obtained by following the saddlenode and period-doubling bifurcations, respectively. The intersections of the vertical dashed lines and 
(a)
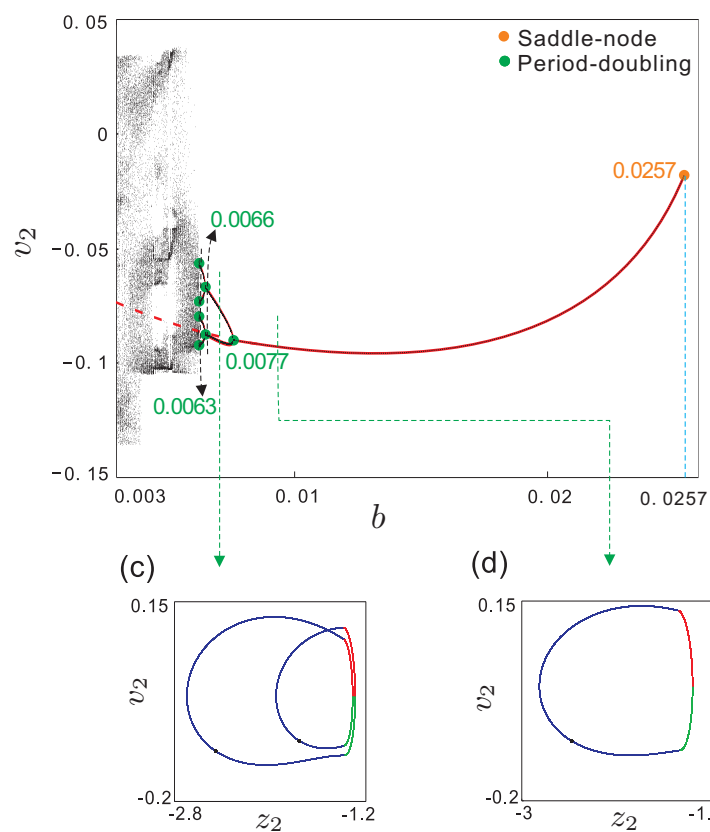

(g)

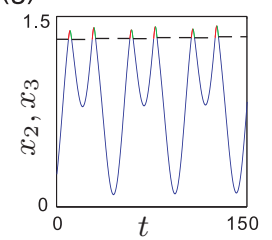

(h) (b)
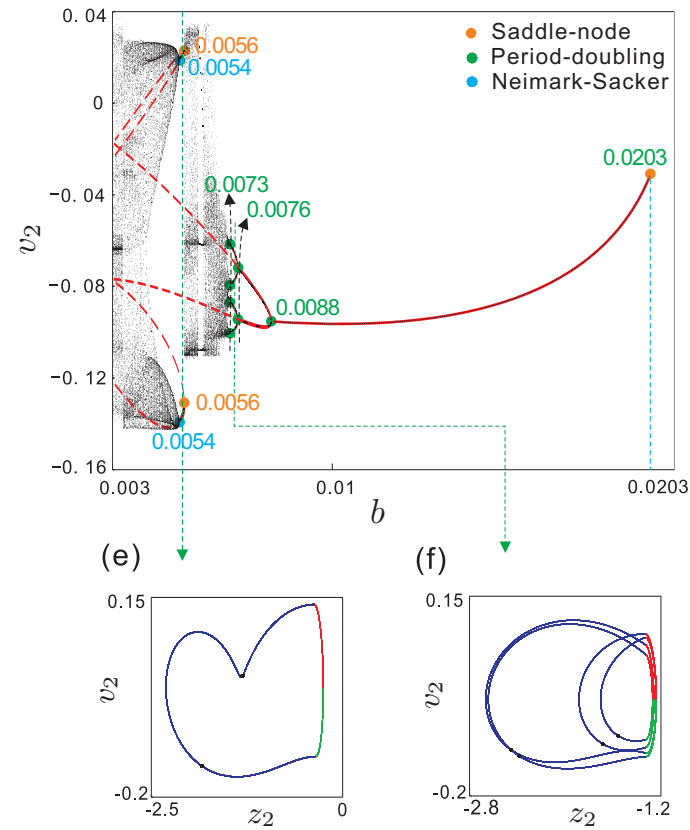

(i)

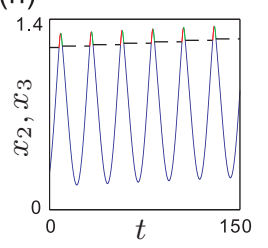

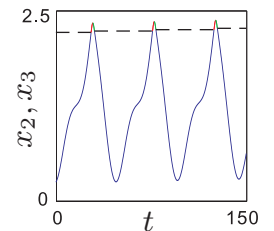

(j)

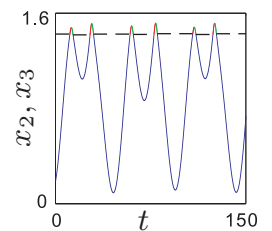

Figure 3.4: Bifurcation diagrams of the nondimensional static force for the higher order drifting oscillator with the (a) linear and (b) nonlinear bit-rock impact models, where the nondimensional parameters for plotting subplot (a) are $a=0.0130$, $b \in[0.003,0.0257], \xi=0.0172, \alpha=0.0133, \beta=0.0033, g=0.02, \omega=0.2353, n_{1}=1, n_{u}=1$, and $\kappa=1.1706$, and the equivalent parameters for plotting subplot (b) are $a=0.0159, b \in[0.003,0.0203], \xi=0.0188, \alpha=0.0133, \beta=0.0040$, $g=0.02, \omega=0.2595, n_{1}=0.86, n_{u}=1.31$, and $\kappa=3.1815$. The results of numerical integration are shown by black dots, while the stable and unstable branches obtained by numerical continuation are denoted by solid and dashed red lines. Subplots (c)-(f) and (g)-(j) display the phase trajectories and time series of the periodic responses, respectively.

the bifurcation curves are the starting points of these two-parameter continuations, which correspond to the bifurcations presented in Fig. 3.3 and Fig. 3.4.

For the linear interaction model, the results of the two-parameter continuations with respect to $(\omega, a)$ and $(\omega, b)$ are shown in Fig. $3.5(\mathrm{a})$ and $(\mathrm{b})$, and the results for the nonlinear one are shown in Fig. 3.5 (c) and (d), where purple dots indicate the grazing points that terminate two-parameter continuations. It is worth noting that at these points, the bit-rock impact regime is changed between one impact and multiple impacts. As demonstrated in subplots (e) and (g), when the grazing points approach, the nocontact phase disappears gradually, and the bit experiences the loading and unloading phases iteratively. 
As can be seen in Fig. 3.5, the structures of the corresponding two-parameter curves are similar. Comparing subplots (a) and (c), the two-parameter curve of saddle-node bifurcation (orange) for the nonlinear model exists in a larger parameter range than the curve obtained by the linear model. For the two-parameter curve of period-doubling bifurcation (green), the nonlinear model exists until ( $\omega=$ 2.4343, $a=0.0420)$ as $\omega$ increases, while the one for the linear model terminates at $(\omega=2.0530, a=$ 0.0310). When $\omega$ decreases, the green curve for the nonlinear model ends at ( $\omega=0.1276, a=0.3970)$, but the one for the linear model can extend further. Comparing subplots (b) and (d), the saddle-node curve (orange) for the nonlinear model ends at $(\omega=1.1585, a=0.0841)$ as $\omega$ increases, while the curve for the linear model can extend further.

In addition, an isola of period-doubling bifurcations [31-33] is observed in both Fig. 3.5 (b) and (d). There are two turning points (red dots) on the isola, which define the interval of existence of the isola. An illustration of the vibration conditions corresponding to the bottom and upper branches of the isola are shown in subplots (f) and (h), respectively. As presented in Fig. 3.5 (f), the bit experiences a long no-contact period followed by a short loading and unloading period. For the upper branch, a short no-contact period accompanying with a long loading and unloading period is recorded in Fig. 3.5 (h).

\section{Rate of penetration}

The main purpose of studying the higher order drifting oscillator with the linear and nonlinear impact models is to investigate their rates of penetration for improving drilling efficiency. According to [5], the stable period-one response with one impact per period of excitation provides the best performance of vibro-impact drilling in terms of the rate of penetration (ROP), which is calculated as,

$$
R O P=\frac{1}{T}\left[z_{f}-z_{p}\right]
$$

where $T$ is the excitation period. Therefore, the ROPs of the stable period-one responses observed in Fig. 3.1-3.4 will be compared in this section.

Fig. 4.1 presents all the calculated ROPs for the higher order drifting oscillator with the linear and 
(a)

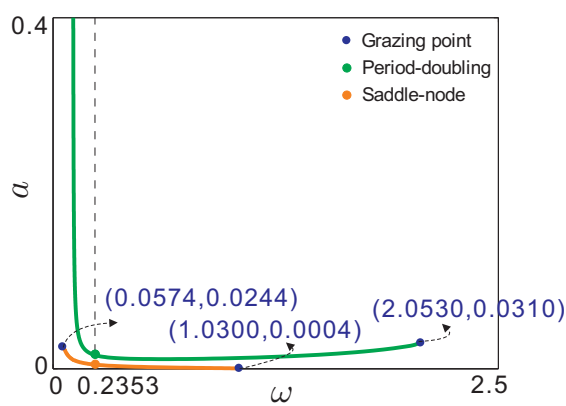

(c)

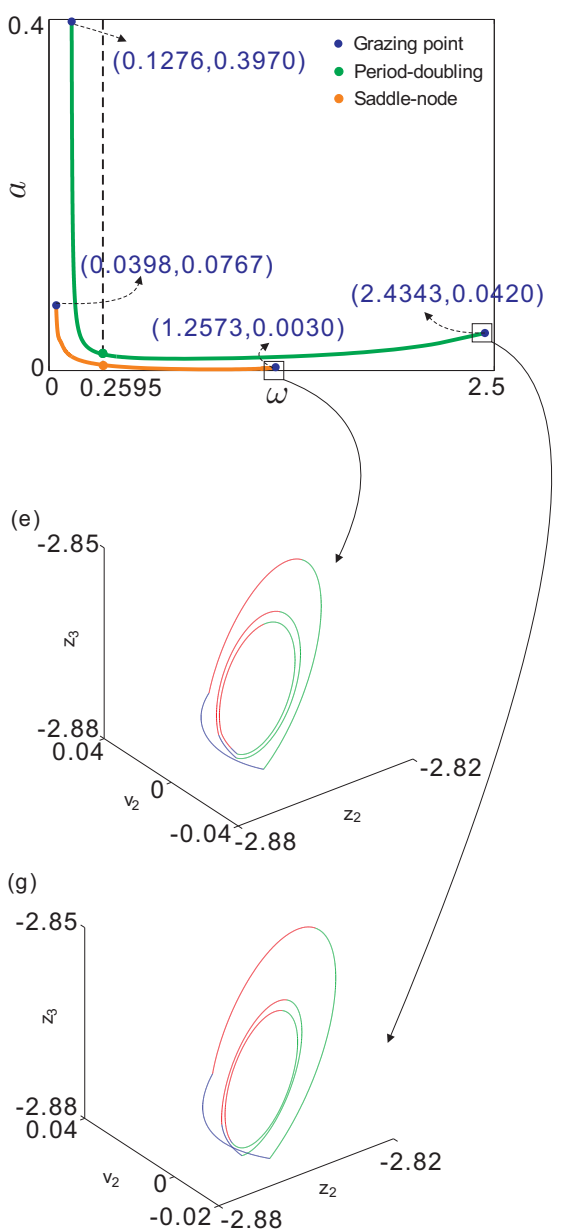

(b)

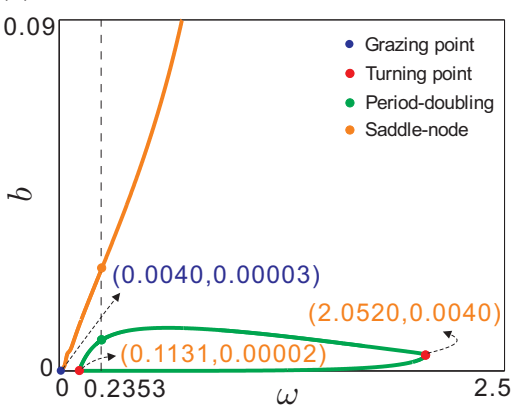

(d)

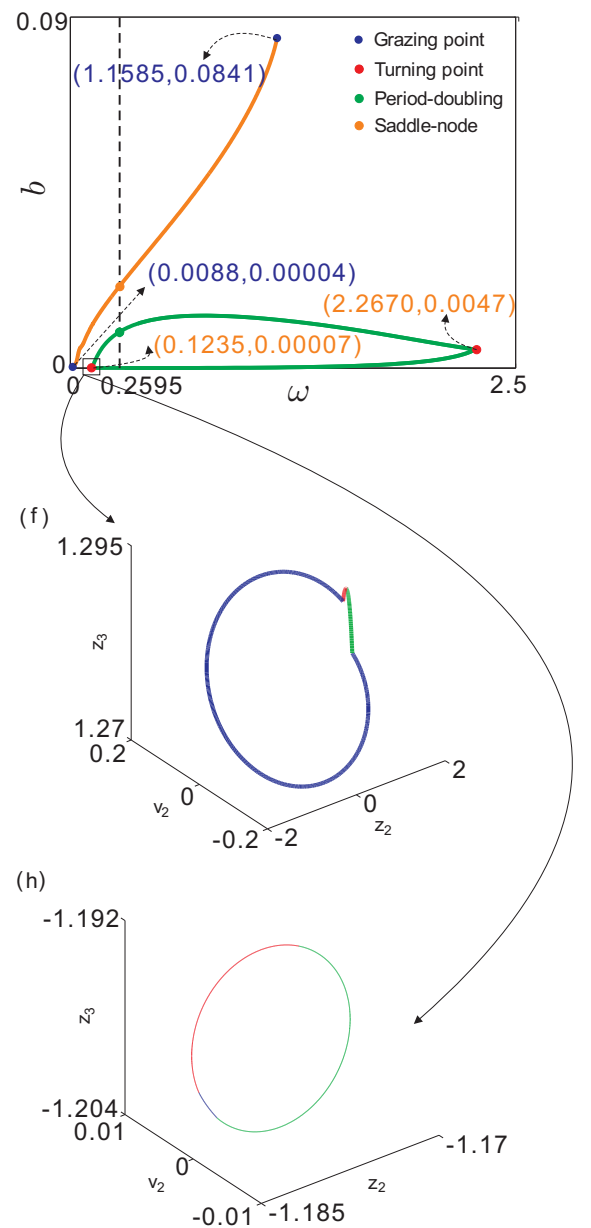

Figure 3.5: Two-parameter continuations for the $(\mathrm{a}, \mathrm{b})$ linear and $(\mathrm{c}, \mathrm{d})$ nonlinear models, the constant nondimensional parameters for subplots $(\mathrm{a}, \mathrm{b})$ are $\xi=0.0172, \alpha=0.0133, \beta=0.0033, g=0.02, n_{1}=1, n_{u}=1$, and $\kappa=1.1706$, and the parameters for subplots (c, d) are $\xi=0.0188, \alpha=0.0133, \beta=0.0040, g=0.02, n_{1}=0.86, n_{u}=1.31$, and $\kappa=3.1815$. Green and orange curves indicate period-doubling and saddle-node bifurcations, respectively. Green and orange dots are the bifurcation points shown in Figs. 3.3 and 3.4, and these dots are also the starting points of the two-parameter continuations. Purple dots are the grazing points at where the two-parameter continuations terminate. Red dots are the turning points of the isola for period-doubling bifurcation [31]. Subplots (e) and (g) display the variations of the vibration conditions when the grazing points approach. Subplot (f) and (h) display the vibration conditions of the bottom and upper branches of the isola for period-doubling bifurcations, respectively. 
nonlinear impact models by varying different control parameters, $\omega, a$, and $b$. As can be seen from Fig. 4.1 (a) and (b), the stable period-one response exists in $\omega \in[0.1449,0.3064]$ for the linear model, and the stable period-one response for the nonlinear model exists in $\omega \in[0.1270,0.3795]$. The maximum ROP, $R O P=6.07 \times 10^{-4}$, for the linear model achieves at $\omega=0.2$, and the maximum ROP for the nonlinear model obtained at $\omega=0.1270$ is $R O P=19.57 \times 10^{-4}$. According to Fig. 3.3 (c) and (d), the stable periodone responses for the linear and nonlinear impact models are $a \in[0.0047,0.0157]$ and $a \in[0.0075,0.0213]$, respectively. As the amplitude of excitation increases, the ROPs for both models increase, and the maximum ROP is achieved at $a=0.0157$ for the linear model and $a=0.0213$ for the nonlinear one. Similar trend can be observed from Fig. 3.4 (e) and (f) when static force increases. The stable periodone responses are recorded for $b \in[0.0077,0.0257]$ for the linear model and $b \in[0.0088,0.0203]$ for the nonlinear model with the maximum ROP obtained at $b=0.00257$ and $b=0.0203$, respectively.

Comparing the above ROPs, it can be found that the ROP for the nonlinear impact model is more sensitive to the frequency of excitation as a small variation of the frequency will cause a significant reduction on ROP. On the other hand, the amplitude of excitation and the static force have more effect on ROP when the nonlinear impact model is considered for vibro-impact drilling. This is evaluated from the gradients of the stable period-one curves in Fig. 3.4 (c)-(f). 
(a)

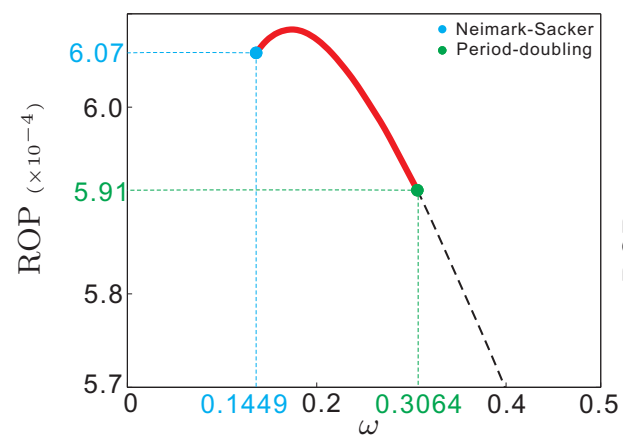

(c)

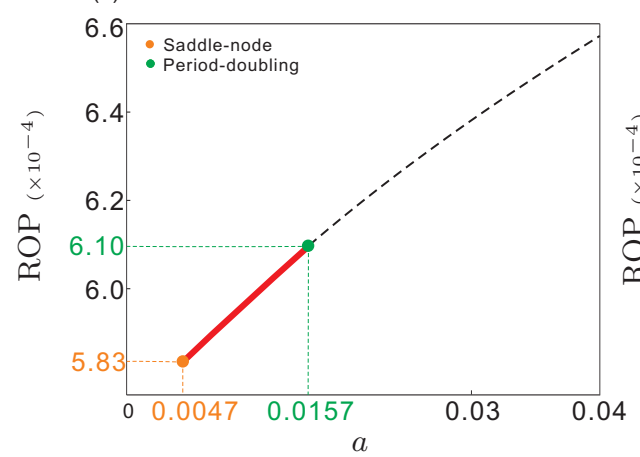

(e)

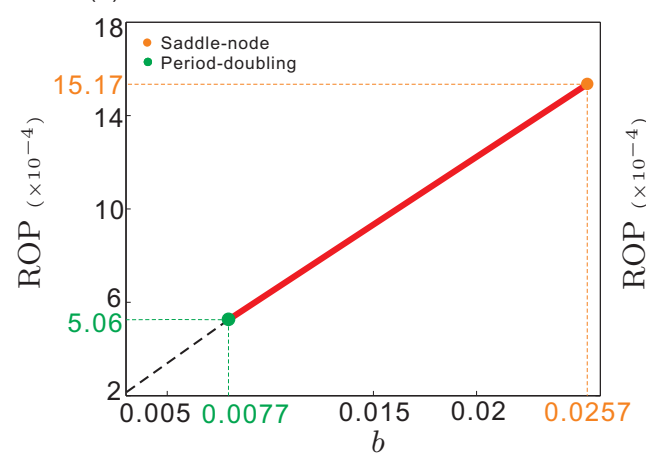

(b)

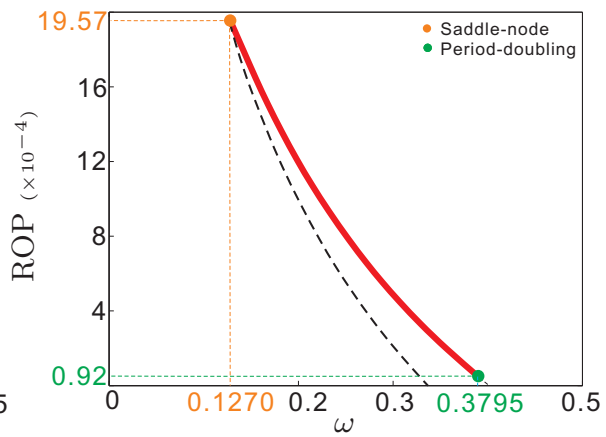

(d)

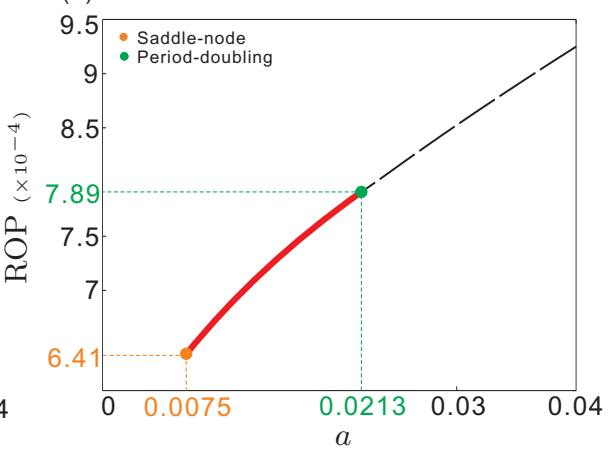

(f)

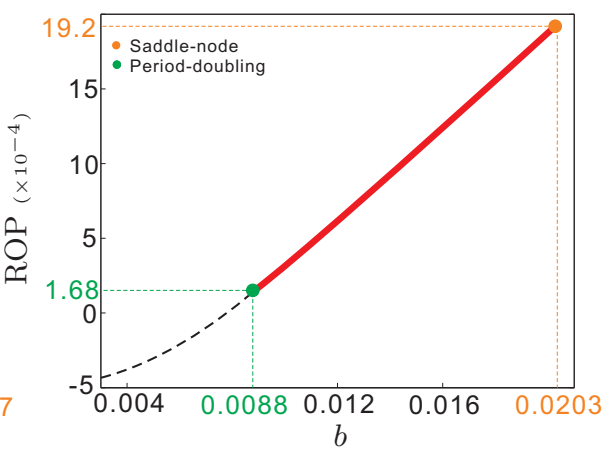

Figure 4.1: Variations of penetration rates for period-one one-impact responses. Subplots (a) and (b) show the penetration rates against frequency for the linear and nonlinear models, and their parameters are the same as those applied in Fig. 3.1 and Fig. 3.2, respectively. Subplots (c) and (d) show the penetration rates against amplitude for the linear and nonlinear models, and the corresponding parameters are the same as those applied in Fig. 3.3. Subplots (e) and (f) show the penetration rates against static force for the linear and nonlinear models, and the corresponding parameters are the same as those applied in Fig. 3.4. The solid red segments represent the stable branches, while the dashed black segments show the obtained unstable branches.

\section{Concluding remarks}

This work presented a detailed numerical study of a higher order drifting oscillator that mimics the dynamics observed in vibro-impact drilling processes. Our main concern was to study the system response when the bit-rock interaction is modeled via linear and nonlinear physical laws. Our investigation 
proposed a numerical approach based on direct numerical integration via a newly developed MATLABbased computational tool, ABESPOL [10], and based on path-following methods implemented via a software package for continuation and bifurcation analysis, COCO [11]. Our numerical study considered the excitation frequency, excitation amplitude and the static force as the main control parameters, and the rate of penetration (ROP) was used as solution measure in order to assess the performance of the system for the linear and nonlinear bit-rock impact models.

The one-parameter bifurcation study revealed the presence of saddle-node, period-doubling and NeimarkSacker bifurcations of limit cycles. When the frequency of excitation varies, a parameter window was identified where three co-existing attractors can be observed, when the linear bit-rock interaction model is considered. This multistability phenomenon, however, disappears when the nonlinear law is implemented. On the other hand, for both models a parameter window of chaotic motion was observed, however, for the nonlinear case the window is smaller in comparison to the one found for the linear bit-rock interaction law. Another relevant difference concerns the range of stability for the period-one response (with one impact per period), which is larger for the nonlinear case. Moreover, the bifurcation structure obtained by varying the excitation amplitude was qualitatively similar for the linear and nonlinear bit-rock interaction laws. A significant difference, however, could be observed in the stability range for the period-one motion, which was smaller for the linear case. An analogous situation was encountered when the static force was considered as the bifurcation parameter. Once again, the bifurcation structure was similar for the linear and nonlinear bit-rock interaction models, but the interval of stability was in this case larger for the linear law.

Our study also presented the two-parameter continuation of some of the codimension-one bifurcations detected in the bifurcation analysis. As could be observed from Fig. 3.5, the resulting two-parameter bifurcation picture showed clear similarities in qualitative terms. The precise numerical values and parameter ranges, however, present differences which are nevertheless not significant. A remarkable common feature of the bifurcation picture was the presence of an isola of period-doubling bifurcations of limit cycles in the $\omega-b$ plane, which indicates that this dynamical phenomenon is robust and has its roots in the main structure of the mathematical model. Similarly, the transition from periodic regimes 
with one impact per period to periodic motions with multiple impacts via grazing phenomena could be detected for both linear and nonlinear bit-rock interaction models.

Another feature that was investigated in the present work was the behavior of the ROP as the system parameters are perturbed. It was found that for the nonlinear model the ROP is more sensitive to variations of the frequency of excitation, as small changes in this parameter produced large fluctuations in the ROP, which can also lead to significant drops in the drilling speed. Similarly, the amplitude of excitation and the static force presented a stronger influence on the ROP when the nonlinear law is used. Therefore, for the parameter ranges considered, our numerical investigation indicates that the linear and nonlinear bit-rock impact models can produce qualitatively similar system behavior, provided the higher order drifting oscillator operates under low frequencies of excitation, small excitation amplitude or small static forces. In these cases, no relevant differences can be observed during the system operation. However, if the parameter values do not meet these conditions, the dynamics of the higher order drifting oscillator can differ significantly, and further studies should be conducted in order to determine what type of bit-rock interaction model better describes the vibro-impact drilling process.

\section{Acknowledgements}

This paper is supported by the National Key Basic Research Program of China (973 Program) (Grant No. 2015CB251206), and the National Natural Science Foundation of China (No. 51775038).

\section{References}

[1] O.K. Ajibose, M. Wiercigroch, E. Pavlovskaia, A.R. Akisanya, "Global and local dynamics of drifting oscillator for different contact force models," International Journal of Nonlinear Mechanics, vol. 45, pp. 850-858, 2010.

[2] O.K. Ajibose, M. Wiercigroch, E. Pavlovskaia, A.R. Akisanya, G. Károlyi, "Drifting impact oscillator with a new model of the progression phase," Journal of Applied Mechanics, vol. 79, p. 061007, 2012.

[3] O.K. Ajibose, M. Wiercigroch, A.R. Akisanya, "Experimental studies of the resultant contact forces in drillbit-rock interaction," International Journal of Mechanical Sciences, vol. 91, pp. 3-11, 2015. 
[4] A.M. Krivtsov, M. Wiercigroch, "Penetration rate prediction for percussive drilling via dry friction model," Chaos, Solitons \& Fractals, vol. 11, no. 15, pp. 2479-2485, 2000.

[5] E. Pavlovskaia, D.C. Hendry, M. Wiercigroch, "Modelling of high frequency vibro-impact drilling," International Journal of Mechanical Sciences, vol. 91, pp. 110-119, 2005.

[6] E. Pavlovskaia, M. Wiercigroch, "Analytical drift reconstruction for visco-elastic impact oscillator operating in periodic and chaotic regimes," Chaos, Solitons 83 Fractals, vol. 19, pp. 151-161, 2004.

[7] J. Páez Chávez, E. Pavlovskaia, M. Wiercigroch, "Bifurcation analysis of a piecewise-linear impact oscillator with drift," Nonlinear Dynamics, vol. 77, pp. 213-227, 2014.

[8] M. Liao, J. Ing, J. Páez Chávez, M. Wiercigroch, "Bifurcation techniques for stiffness identification of an impact oscillator," Commun. Nonlinear Sci. Numer. Simul., vol. 41, pp. 19-31, 2016.

[9] M. Liao, J. Ing, M. Sayah, M. Wiercigroch, "Dynamic method of stiffness identification in impacting systems for percussive drilling applications," Mechanical Systems and Signal Processing, vol. 80, pp. $224-244,2016$.

[10] A.S.E. Chong, Y. Yue, E. Pavlovskaia, M. Wiercigroch, "Global dynamics of a harmonically excited oscillator with a play: Numerical studies," International Journal of Nonlinear Mechanics, vol. 94, pp. 98-108, 2017.

[11] H. Dankowicz, F. Schilder, Recipes for Continuation. Philadelphia, USA: Computational Science \& Engineering, 2013.

[12] L.L. Mishnaevsky, "Physical mechanism of hard rock fragmentation under mechanical loading a review," Int J. Rock Mech Min Sci., vol. 32, no. 8, pp. 763-765, 1995.

[13] N.G.W. Cooka, M. Hooda, F. Tsai, "Observations of crack growth in hard rock loaded by an indenter," Int.J. Rock Mech. Min. Sci. \& Geomech. Abstr., vol. 21, no. 2, pp. 97-107, 1984.

[14] L. Půst, F. Peterka, "Impact oscillator with Hertz's model of contact," Meccanica, vol. 38, no. 1, pp. 99-114, 2003. 
[15] T. Hatada, T. Kobori, M. Ishida, N. Niwa, "Dynamic analysis of structures with Maxwell model," Earthquake Engineering and Structural Dynamics, vol. 29, no. 2, pp. 159-176, 2000.

[16] E. Pavlovskaia, M. Wiercigroch, C. Grebogi, "Modeling of an impact system with a drift," Physical Review E, vol. 64, no. 5, p. 056224, 2001.

[17] M.S. Sazidy, D.G. Rideout, S.D. Butt, F. Arvani, "Modeling percussive drilling performance using simulated visco-elasto-plastic rock medium," in 44th US Rock Mechanics Symposium and 5th U.S.Canada Rock Mechanics Symposium, (Salt Lake City, UT, USA), 2010.

[18] Y. Kovalyshen, "Self-excited axial vibrations of a drilling assembly: modeling and experimental investigation," in 47th US Rock Mechanics/Geomechanics Symposium, (San Francisco, CA, USA), 2013.

[19] G. Han, M.S. Bruno, M.B. Dusseault, "Dynamically modelling rock failure in percussion drilling," in Symposium on Rock Mechanics (USRMS), (Anchorage, Alaska), 2005.

[20] G. Han, M.S. Bruno, "Percussion drilling: from lab tests to dynamic modeling," in International Oil 83 Gas Conference and Exhibition in China, (Beijing, China), 2006.

[21] G. Han, M.S. Bruno, T. Grant, "Lab investigations of percussion drilling: from single impact to full scale fluid hammer," in The 41st U.S. Symposium on Rock Mechanics (USRMS), (Golden, Colorado), 2006.

[22] CK. Loong, J. Wojewoda, M. Wiercigroch, "Design of load cell for measurement ultrasonic percussive drilling forces," Proceedings of Institution of Mechanical Engineers - Part C, vol. 215, no. 3, pp. 965$972,2001$.

[23] M. Wiercigroch, J. Wojewoda, A.M. Krivtsov, "Dynamics of ultrasonic percussive drilling of hard rocks," Journal of Sound and Vibration, vol. 280, pp. 739-757, 2005.

[24] M. Wiercigroch, R.D. Neilson, M.A. Player, "Material removal rate prediction for ultrasonic drilling of hard materials using impact oscillators approach," Physics Letters A, vol. 259, no. 2, pp. 91-96, 1999. 
[25] Q. Cao, M. Wiercigroch, E. Pavlovskaia, S. Yang, "Bifurcations and the penetrating rate analysis of a model for percussive drilling," Acta Mechanica Sinica, vol. 26, no. 3, pp. 467-475, 2010.

[26] P. Thota, H. Dankowicz, "TC-HAT: A novel toolbox for the continuation of periodic trajectories in hybrid dynamical systems," SIAM J. Appl. Dyn. Sys., vol. 7, no. 4, pp. 1283-1322, 2008.

[27] M. Liao, Y. Su, Y. Zhou, "Oscillation reconstruction and bifurcation analysis of a drillbitrock vibroimpact system," International Journal of Bifurcation and Chaos, vol. 27, no. 1, p. 1750013, 2017.

[28] H. Jiang, A.S.E Chong, Y. Ueda, M. Wiercigroch, "Grazing-induced bifurcations in impact oscillators with elastic and rigid constraints," International Journal of Mechanical Sciences, vol. 127, pp. 204$214,2017$.

[29] A.S.E. Chong, P. Brzeski, M. Wiercigroch, P. Perlikowski, "Path following bifurcation analysis of the church bell: autonomous hybrid dynamical system," Journal of Computational and Nonlinear Dynamics, vol. 12, no. 6, pp. 1-8, 2017.

[30] M. Sayah, Nonlinear time series analysis applied to resonance enhanced drilling. University of Aberdeen: PhD thesis, 2015.

[31] C. Mayol, M.A. Natiello, M.G. Zimmermann, "Resonance structure in a weakly detuned laser with injected signal," International Journal of Bifurcation and Chaos, vol. 11, no. 10, pp. 2587-2605, 2001.

[32] D.David, K.B. Herbert, M.J. Bernard, R.L. Edward, "On the birth of isolas," SIAM Journal on Applied Mathematics, vol. 42, no. 5, pp. 956-963, 1982.

[33] T. Kupper, H.D. Mittelmann, H. Weber, "Numerical methods for bifurcation problems," in International Series of Numerical Mathematics, (Dortmund, Germany), 1983. 